\title{
CORRESPONDENCE
}

The Joint Editors,

The Journal of the Institute of

I2 December 1949

Actuaries Students' Society

\section{A Proof of Certain Statistical Distributions}

Sirs,

Dr J. Wishart, following an admirable lecture(x), recently gave your readers a simple proof of certain fundamental theorems in mathematical statistics $(2,3)$.

What I believe to be an even simpler proof of some of the results, based on a method given by Prof. J. F. Steffensen (4), may be stated as follows:

$x_{i}, i=\mathrm{I}$ to $n$, are $n$ independent observations from a normal distribution with mean $\xi$ and variance $\sigma^{2}$, so that the probability that $x_{1} x_{2} \ldots x_{n}$ fall within $d x_{1} d x_{2} \ldots d x_{n}$ is given by

$$
\begin{aligned}
& p\left(x_{1} \ldots x_{n}\right) d x_{1} \ldots d x_{n} \\
& \quad=\left(2 \pi \sigma^{2}\right)^{-\frac{1}{2} n} \exp \left[-\frac{1}{2}\left(\sum_{i=1}^{n}\left(x_{i}-\xi\right)^{2}\right\} / \sigma^{2}\right] d x_{1} \ldots d x_{n} .
\end{aligned}
$$

Note that $\quad \sum_{i=1}^{n}\left(x_{i}-\xi\right)^{2}=\sum_{i=1}^{n}\left(x_{i}-\bar{x}\right)^{2}+n(\bar{x}-\xi)^{2}$, where $\bar{x}=\sum_{i=1}^{n} x_{i} / n$.

Let us make the transformation

$$
x_{i}=z+u_{i} \sqrt{ } \mathrm{S} \quad(i=\mathrm{I} \text { to } n)
$$

subject to

$$
\sum_{i=1}^{n} u_{i}=0 \quad \text { and } \quad \sum_{i=1}^{n} u_{i}^{2}=1 \text {. }
$$

Summing the $n$ equations shows that

$$
z=\sum_{i=1}^{n} x_{i} / n=\bar{x}
$$


since $\sum_{i=1}^{n} u_{i}=0$, and summing the $\left(x_{i}-z\right)^{2}$ shows that

since

$$
\begin{aligned}
\mathrm{S}= & \sum_{i=1}^{n}\left(x_{i}-\bar{x}\right)^{2}, \\
& \sum_{i=1}^{n} u_{i}^{2}=\mathrm{I} .
\end{aligned}
$$

Further, since two equations hold between the $u_{1}$, any two of them, say $u_{n-1}$ and $u_{n}$, can in theory be expressed in terms of the remaining $u_{i}$.

We are thus transforming from $n$ functionally independent variables $x_{i}$ to $n$ functionally independent variables $\bar{x}, \mathrm{~S}, u_{1} \ldots u_{n-2}$. The Jacobian of the transformation is then $\partial\left(x_{1} \ldots x_{n}\right) / \partial\left(\bar{x} \mathrm{~S} u_{1} \ldots u_{n-2}\right)$, which is

$$
\left|\begin{array}{cccccccc}
\mathrm{I} & \frac{u_{1}}{2 \sqrt{\mathrm{S}}} & \sqrt{ } \mathrm{S} & \circ & \circ & : & : & \circ \\
\mathrm{I} & \frac{u_{2}}{2 \sqrt{\mathrm{S}}} & \circ & \sqrt{ } \mathrm{S} & \circ & : & : & \circ \\
: & : & : & : & : & : & : & : \\
: & : & : & : & : & : & : & : \\
\text { I } & \frac{u_{n-2}}{2 \sqrt{ }} & \circ & \circ & \circ & : & : & \sqrt{\mathrm{S}} \\
\mathrm{I} & \frac{u_{n-1}}{2 \sqrt{ } \mathrm{S}} & \sqrt{ } \mathrm{S} \frac{\partial u_{n-1}}{\partial u_{1}} & : & : & : & : & \sqrt{ } \mathrm{S} \frac{\partial u_{n-1}}{\partial u_{n-2}} \\
\text { I } & \frac{u_{n}}{2 \sqrt{S}} & \sqrt{ } \mathrm{S} \frac{\partial u_{n}}{\partial u_{1}} & : & : & : & : & \sqrt{ } \mathrm{S} \frac{\partial u_{n}}{\partial u_{n-2}}
\end{array}\right|
$$

This formidable looking affair immediately factorizes into

$$
\begin{array}{|c|cccccccc}
\mathrm{S}(n-3) & \mathrm{I} & \frac{1}{2} u_{1} & \mathrm{I} & \circ & \circ & : & : & 0 \\
\mathrm{I} & \frac{1}{2} u_{2} & \circ & \mathrm{I} & \circ & : & : & 0 \\
: & : & : & : & : & : & : & : \\
: & : & : & : & : & : & : & : \\
\mathrm{I} & \frac{1}{2} u_{n-2} & \circ & \circ & \circ & : & : & \mathrm{I} \\
\mathrm{I} & \frac{1}{2} u_{n-1} & \frac{\partial u_{n-1}}{\partial u_{1}} & : & : & : & : & \frac{\partial u_{n-1}}{\partial u_{n-2}} \\
& & \frac{\partial u_{n}}{\partial u_{1}} & : & : & : & : & \frac{\partial u_{n}}{\partial u_{n-2}}
\end{array}
$$


and all we need note about this determinant is that it is a function of the $u_{i}$ only, say $f\left(u_{1} \ldots u_{n-2}\right)$, and does not contain $\bar{x}$ or $\mathrm{S}$.

Performing the transformation, remembering that $d x_{1} d x_{2} \ldots d x_{n}$ transforms into $|\mathrm{J}| d \mathrm{~S} d \bar{x} d u_{1} \ldots d u_{n-2}$, we find that the probability that $\mathrm{S}, \bar{x}, u_{1} \ldots u_{n-2}$ fall within $d \mathrm{~S} d \bar{x} d u_{1} \ldots d u_{n-2}$ is given by

$p\left(\mathrm{~S}, \bar{x}, u_{1} \ldots u_{n-2}\right) d \mathrm{~S} d \bar{x} d u_{1} \ldots d u_{n-2}$

$$
=c \mathrm{~S}^{\frac{1}{(2 n-3)}} \exp \left[-\frac{1}{2}\left\{\mathrm{~S}+n(\bar{x}-\xi)^{2}\right\} / \sigma^{2}\right] f\left(u_{1} \ldots u_{n-2}\right) d \mathrm{~S} d \bar{x} d u_{1} \ldots d u_{n-2},
$$

where $c$ is a constant.

This factorizes at sight and shows that

$$
\begin{aligned}
& p(\mathrm{~S}) d \mathrm{~S}=c_{1} \mathrm{~S}^{\frac{1}{(n-3)}} \exp \left(-\frac{1}{2} \mathrm{~S} / \sigma^{2}\right) d \mathrm{~S}, \\
& p(\bar{x}) d \bar{x}=c_{2} \exp \left\{-\frac{1}{2} n(\bar{x}-\xi)^{2} / \sigma^{2}\right\} d \bar{x} \\
& p\left(u_{1} \ldots u_{n-2}\right) d u_{1} \ldots d u_{n-2}=c_{3} f\left(u_{1} u_{2} \ldots u_{n-2}\right) d u_{1} d u_{2} \ldots d u_{n-2} .
\end{aligned}
$$

It also shows that $\mathrm{S}, \bar{x}$ and the $u_{i}$ taken as a group are stochastically independent. (Among themselves the $u_{i}$ are correlated; but this is a fact without interest to us here.) $c_{1}$ and $c_{2}$ are rapidly obtained from

$$
\int_{0}^{\infty} p(\mathrm{~S}) d \mathrm{~S}=\int_{-\infty}^{\infty} p(\bar{x}) d \bar{x}=\mathrm{I} .
$$

The $u_{i}$ we cheerfully ignore since we are not interested in them, and the method has yielded the two distributions that we do want in a delightfully 'sudden' manner. The method can be applied again, with a slight modification, in the first part of Dr Wishart's third paper(3), but I do not wish to use up any more of your valuable space.

\section{Yours faithfully,}

University of the Witwatersrand

J. E. KERRICH

\section{REFERENCES}

(I) WISHaRT, J. (1947). The variance ratio test in statistics. J.S.S. Vol. vi, p. 172.

(2) Wishart, J. (1947). Proof of the distribution of $\chi^{2}$, of the estimate of the variance and of the variance ratio. Y.S.S. Vol. viI, p. 98.

(3) Wishart, J. (1948). Tests of significance in the simple regression problem. F.S.S. Vol. viII, p. 38 .

(4) Steffensen, J. F. (1936). Free functions and the Student-Fisher theorem. Skand. AktuarTidskr. Vol. xIx, p. I08.

[The method used by our correspondent may be compared with $R$. A. Fisher's 'geometrical' method, of which it appears to be the analytical equivalent. The subject is discussed in Chapter 1o of M. G. Kendall's Advanced Theory of Statistics.—Eds. Y.S.S.] 